\title{
A Bibliography on Hinduism
}

\section{J. F. SPROCKHOFF (KIEL) AND J. GONDA (UTRECHT)}

Considering the growth of specialization and the increasing number of publications in the various fields of Indology, research on Hinduism already stands in need of a bibliog. raphy exclusively concerning this particular field, where it will serve scholars as a guide and tool. Such research aids are already available in the fields of Veda, Buddhism, Indian archaeology, and anthropology. The lack of a similar tool for the study of Hinduism constitutes an even more serious shortcoming in our Indological equipment. With a view to meeting this need, a detailed plan was drafted in the summer of 1962 at the Institute of Oriental Studies (Utrecht) by J. F. Sprockhoff under the direction of J. Gonda. The general idea of this plan and the detailed report on the ways and means of its implementation have met with favourable reactions from colleagues to whom the plan has been submitted so far.

The bibliography is to be, like the already existing ones mentioned above, a critical one. It is further intended to encompass materials and studies relating to religion, philosophy, law, and customs-each in its widest sense-as well as all other documentation on the cultural history of India as far as it has source value for the study of Hinduism. This means that not only Sanskrit sources but also the vernacular sources will be registered. As regards the secondary literature of the last hundred years, it is to be taken into account that the results of research on Hinduism are no longer being made available exclusively through the medium of western European languages. It seems clear therefore that the bibliography project can be implemented only through international cooperation.

So far we are not aware of a similar project contemplated or put into operation elsewhere. It will be welcomed if any such plans could be made known and coordinated with the project planned at Utrecht. Through the present communication, scholars in this field are invited to extend their help and co-operation. Requests for further information concerning method, organization, and planning of the bibliography, as well as offers of co-operation under whatever form, will be welcomed at the following address:

\section{J. GONDA \\ c/o Institute of Oriental Studies \\ Nobelstraat 2 B \\ Utrecht-Netherlands.}

It is hoped that work on the project can be started in 1963 . Scholars in this field are therefore kindly requested to send offprints and review copies, carrying the usual data of date and place of publication, to the above address.

Further memoranda on the progress of the project will from time to time be made available in the columns of Indological journals.

\section{Annual Report of the Association for Asian Studies, 1962-1963}

During its Philadelphia convention in $196_{3}$, the Association for Asian Studies observed its fifteenth anniversary. As the founding fathers can testify, the activities of the first year of the society's life were a marked contrast in scope and significance to those of today. Established as the Far Eastern Association in 1948, the organization changed its name to the Association for Asian Studies in 1957 as a consequence of broadening its geographic area of interest to include South Asia. Membership in the American Council of Learned Societies and participation along with the American Oriental Society in the International Congress of Orientalists are indicative of its stature. In almost every phase of activity, 